Marcin Szyszkowski*, Department of Mathematics, West Virginia University, Morgantown, WV 26506-6310, and Department of Mathematics, Gdańsk

University, Wita Stwosza 57, Gdańsk, Poland. e-mail: Marcin@math.wvu.edu

\title{
SYMMETRICALLY CONTINUOUS FUNCTIONS ON VARIOUS SUBSETS OF THE REAL LINE
}

\begin{abstract}
We define symmetric continuity for functions defined on arbitrary subsets of $\mathbb{R}$. The main result is that when a symmetrically continuous function is defined on a measurable set (a set with the Baire property), then it is continuous almost everywhere (on a residual set, respectively). This generalizes the known result for functions defined on the whole real line.
\end{abstract}

\section{Introduction}

The study of symmetric functions and symmetrically continuous functions stems from the theory of trigonometric series. In theory of trigonometric series, we study functions defined on various subsets of $\mathbb{R}$, while symmetrically continuous functions have been studied so far only on the whole real line or on intervals. We examine basic properties of symmetrically continuous functions defined on arbitrary subsets of $\mathbb{R}$.

We say that a function $f: \mathbb{R} \rightarrow \mathbb{R}$ is symmetrically continuous at a point $x \in \mathbb{R}$ if

$$
\forall \varepsilon>0 \exists \delta>0 \forall|h|<\delta \quad|f(x+h)-f(x-h)|<\varepsilon
$$

or, equivalently

$$
\lim _{h \rightarrow 0} f(x+h)-f(x-h)=0 .
$$

We define symmetrical continuity for a function defined on any subset of reals in the following way.

\footnotetext{
Key Words: symmetrically continuous, measurability, Baire property.

Mathematical Reviews subject classification: 26A15

Received by the editors February 16, 1999

* The author wishes to thank Professor K. Ciesielski for fruitful discussion and invaluable help with writing this paper.
} 
Definition 1. Let $A \subset \mathbb{R}$. A function $f: A \rightarrow \mathbb{R}$ is said to be symmetrically continuous at a point $x$ if

$$
\forall \varepsilon>0 \exists \delta>0 \forall|h|<\delta \quad x \pm h \in A \Rightarrow|f(x+h)-f(x-h)|<\varepsilon .
$$

If $f$ is symmetrically continuous at every point of $A$, then we we say that $f$ is symmetrically continuous on $A$ or simply symmetrically continuous.

Because of the implication above, it is possible (and in fact we will often consider such a situation) that $f$ is symmetrically continuous at $x$ only because for every $x+h \in A$ the number $x-h$ is not in $A$. If this happens, then we say that $f$ is vacuously symmetrically continuous at this point. In Section 5 we address the issue when the domain $A$ is symmetric; that is, $x+h \in A$ if and only if $x-h \in A$. Requiring additionally in Definition 1 that the function is defined on a symmetric domain would narrow our considerations and would not improve our results (at least when the domain is measurable or has the Baire property).

Note that we do not need a function $f$ to be defined at a point $x$ to talk about symmetric continuity at $x$. However we will concentrate only on the points from the domain (except in Theorem 2).

Obviously ordinary continuity implies symmetric continuity, but not conversely. Let us give some simple examples. The discontinuous function $f(x)=$ 0 for $x \neq 0$ and $f(0)=1$ is symmetrically continuous everywhere. The function $\cos \left(\frac{1}{x}\right)$ is symmetrically continuous everywhere (including 0 ) but $\sin \left(\frac{1}{x}\right)$ is not symmetrically continuous at 0 .

Example 1. Let $G \subset \mathbb{R}$ be an additive group and $f=\chi_{G}: \mathbb{R} \rightarrow\{0,1\}$ be the characteristic function of $G$. Then $f$ is symmetrically continuous on $G$.

Indeed if $a \in G$, then for any point $s=a+h$ its symmetric reflection about $a$ is $a-h=2 a-s$ and it belongs to $G$ if and only if $s$ does. Outside $G, f$ is symmetrically continuous only at points $x$ for which $2 x \in G$. In particular if $G$ is divisible by 2 , then $f$ is symmetrically discontinuous everywhere outside $G$.

Example 2. Let $G_{1}$ and $G_{2}$ be the additive subgroups in $\mathbb{R}$ with $G_{1} \cap G_{2}=\{0\}$ and let $f: G_{1} \cup G_{2} \rightarrow\{0,1\}=\chi_{G_{1}}$. Then $f$ is symmetrically continuous on its domain.

Just as above, for any $a \in G_{i}, i=1,2$, and $s \in \mathbb{R}$, we have $s \in G_{i}$ if and only if $2 a-s \in G_{i}$. So $f$ is symmetrically continuous at $a$.

Using functions defined on domains smaller than $\mathbb{R}$ we may address an old problem of Marcus to characterize the set of points at which a function $f: \mathbb{R} \rightarrow \mathbb{R}$ may be symmetrically continuous. Without solving this problem we state the following theorem. 
Theorem 2. For any set $A \subset \mathbb{R}$ there is a set $X \subset \mathbb{R}$ and a function $f: X \rightarrow$ $\{0,1\}$ that is symmetrically continuous (vacuously) at $x \in \mathbb{R}$ (not necessarily in $X$ ) if and only if $x \in A$.

This theorem follows immediately from [Sz, lemma 4].

In Section 2 we state the main results about continuity of symmetrically continuous functions. Section 3 consists only of the proofs of theorems from Section 2. In Section 4 we investigate the extension properties of symmetrically continuous functions to bigger domains and the last section is devoted to functions on symmetric domains.

Our notation is standard and follows [Ci] and [Th]. By an interval we always mean a nontrivial interval (i.e., containing more than one point). For different $a, b \in \mathbb{R}$ we write $[a, b]$ for the interval $[\min (a, b), \max (a, b)]$.

We summarize the properties of symmetrically continuous functions defined on subsets of $\mathbb{R}$ in the chart below. We assume here that the function $f: A \rightarrow \mathbb{R}$ is symmetrically continuous. The property " $f$ is extendable" means that $f$ has a symmetrically continuous extension, and " $f$ is almost extendable" means that there is a symmetrically continuous function $F$ defined on a set containing $A$ such that $\{x \in A: f(x) \neq F(x)\}$ has measure zero. The property " $f$ can be extended" indicates that there is an extension of $f$ which is symmetrically continuous in every point of $A$.

The symbol "+" means that the function $f$ has the property in the left column; "-" means that it does not. Directly before the table we include some short explanations. We also included the appropriate examples and theorems for fast reference.

(i) A function $f$ from $A \subset \mathbb{R}$ to $\mathbb{R}$ is measurable (or has the Baire property) if $f$ is the restriction of some measurable $F: \mathbb{R} \rightarrow \mathbb{R}$ (having the Baire property) to the set $A$. This is the same as saying that the preimages under $f$ of open sets are intersections of measurable sets (sets with the Baire property) with $A$. If $f$ is as in Example 2 and $G_{1}$ and $G_{2}$ are of the full outer measure (nowhere meager), then $f$ is nonmeasurable (does not have the Baire property).

(ii) The function $\chi_{(0, \infty)}$ on $\mathbb{R} \backslash\{0\}$ is an example here.

(iii) Take $f$ is as in Example 2 with $G_{1}$ and $G_{2}$ nowhere meager and of the full outer measure.

(iv) Extend $f$ putting 0 on $\mathbb{R} \backslash A$. 


\begin{tabular}{|c|c|c|c|c|}
\hline Domain $A$ is $: \rightarrow$ & $\mathbb{R}$ & any set & $\begin{array}{c}\text { measurable or } \\
\text { with the Baire } \\
\text { property }\end{array}$ & symmetric \\
\hline $\begin{array}{l}\text { measurable or has } \\
\text { the Baire property }\end{array}$ & $\begin{array}{c}\text { Thm. } 4 \\
+\end{array}$ & $\begin{array}{l}\text { (i) } \\
-\end{array}$ & $\begin{array}{c}\text { Cor. } 10 \& 11 \\
+\end{array}$ & (i) Ex.5 \\
\hline $\begin{array}{l}\text { continuous almost } \\
\text { everywhere on } A\end{array}$ & $\begin{array}{l}\text { Cor. } 8 \\
+\end{array}$ & $\begin{array}{c}\text { Ex. } 3 \\
-\end{array}$ & $\begin{array}{c}\text { Cor. } 10 \& 11 \\
+\end{array}$ & $\begin{array}{c}\text { Ex. } 5 \\
-\end{array}$ \\
\hline extendable to $\mathbb{R}$ & $\mathrm{N} / \mathrm{A}$ & $\begin{array}{l}\text { (ii) } \\
-\end{array}$ & $\begin{array}{l}\text { (ii) } \\
-\end{array}$ & $\begin{array}{c}\text { Ex. } 5 \\
-\end{array}$ \\
\hline $\begin{array}{l}\text { extendable to a } \\
\text { measurable set or } \\
\text { a set with the } \\
\text { Baire property }\end{array}$ & $\mathrm{N} / \mathrm{A}$ & Ex. 3 & $\mathrm{~N} / \mathrm{A}$ & Ex. 5 \\
\hline $\begin{array}{c}\text { almost extendable } \\
\text { to } \mathbb{R}\end{array}$ & $\mathrm{N} / \mathrm{A}$ & (ii) & (ii) & $\begin{array}{c}\text { Ex. } 5 \\
-\end{array}$ \\
\hline $\begin{array}{l}\text { almost extendable } \\
\text { to a residual set }\end{array}$ & $\mathrm{N} / \mathrm{A}$ & (iii) & $\begin{array}{c}\text { Cor. } 10 \& 11 \\
+\end{array}$ & $\begin{array}{c}\text { Ex. } 5 \\
-\end{array}$ \\
\hline \multicolumn{5}{|c|}{ extensions preserving only original points of symmetric continuity } \\
\hline $\begin{array}{c}\text { can be extended } \\
\text { to } \mathbb{R}\end{array}$ & $\mathrm{N} / \mathrm{A}$ & $\begin{array}{c}\text { Thm. } 20 \\
-\end{array}$ & Ex. 2 & $\begin{array}{l}\text { (iv) } \\
+\end{array}$ \\
\hline $\begin{array}{l}\text { can be extended } \\
\text { to a residual set }\end{array}$ & $\mathrm{N} / \mathrm{A}$ & $\begin{array}{c}\text { Thm. } 20 \\
-\end{array}$ & $\begin{array}{c}\text { Ex. } 2 \\
-\end{array}$ & $\begin{array}{l}\text { (iv) } \\
+\end{array}$ \\
\hline $\begin{array}{l}\text { can be almost } \\
\text { extended to } \mathbb{R}\end{array}$ & $\mathrm{N} / \mathrm{A}$ & $?$ & $\begin{array}{l}\text { Fact } 19 \\
+\end{array}$ & $\begin{array}{l}\text { (iv) } \\
+\end{array}$ \\
\hline
\end{tabular}

\section{Continuity of Symmetrically Continuous Functions}

It is always a fundamental question how some other type of continuity is related to the ordinary continuity. Although symmetric continuity is weaker than continuity, symmetrically continuous functions on "nice" domains must be continuous at many points. In this section we are going to investigate the set of points of continuity of symmetrically continuous functions defined on arbitrary subsets of $\mathbb{R}$. Our main results here are Theorem 9 and Corollaries 10 and 11, which are strengthenings of earlier known theorems, quoted below, for functions from $\mathbb{R}$ to $\mathbb{R}$. The proofs have been shifted to the next section as they are long and technical.

The first result that we state was published by Stein and Zygmund in 1960.

Theorem 3. (Stein, Zygmund [SZ]) If $f: \mathbb{R} \rightarrow \mathbb{R}$ is symmetrically continuous and measurable, then it is continuous everywhere except on a set of measure 
zero and first category.

Later on Pesin and Preiss showed that the measurability assumption may be dropped.

Theorem 4. (Pesin $[\mathrm{Pe}]$, Preiss $[\mathrm{Pr}])$ If $f: \mathbb{R} \rightarrow \mathbb{R}$ is symmetrically continuous, then $f$ is measurable.

So the obvious corollary says that symmetric continuity implies continuity almost everywhere (in the sense of measure and category).

The category version of that corollary is an old theorem of Fried from 1937 (which uses the method of Charzyński).

Theorem 5. (Fried $[\mathrm{Fr}]$ ) Let $f: \mathbb{R} \rightarrow \mathbb{R}$ be symmetrically continuous on a residual set. Then $f$ is continuous at every point except on a set of the first category.

Stein and Zygmund had also a stronger result saying that measurability and symmetric continuity only on a measurable set also implies continuity almost everywhere on that set.

Theorem 6. (Stein, Zygmund [SZ]) If $f: \mathbb{R} \rightarrow \mathbb{R}$ is measurable and symmetrically continuous on a measurable set $E \subset \mathbb{R}$, then $f$ is continuous almost everywhere (in the sense of measure) on $E$.

One may suspect that here, as before, the measurability assumption is not necessary. Indeed this is the case. It follows from the following theorem of Belna from 1983.

Theorem 7. (Belna $[\mathrm{Be}]$ ) Let $f: \mathbb{R} \rightarrow \mathbb{R}$ be an arbitrary function. Then the set of points at which $f$ is symmetrically continuous but not continuous has inner measure zero and contains no second category set having the Baire property.

Since for every function from $\mathbb{R} \rightarrow \mathbb{R}$ the set of points of continuity is a $G_{\delta}$ set, which is both measurable and has the Baire property, we have the following corollary.

Corollary 8. (Belna $[\mathrm{Be}]$ ) Let $f: \mathbb{R} \rightarrow \mathbb{R}$ be symmetrically continuous on a set $E$ which is measurable (has the Baire property). Then $f$ is continuous at almost every point of $E$ (on a residual subset of $E$ ).

In the corollary above the function $f$ may of course fail to be even measurable on the set $\mathbb{R} \backslash E$. This raises a question whether we need at all the 
assumption that the function is defined on the set $\mathbb{R} \backslash E$. The answer is not obvious since the proofs of all the theorems mentioned above use heavily the fact that the function is defined everywhere on $\mathbb{R}$. However, it is indeed enough that the function $f$ is defined only on the set $E$. It follows from the next theorem, which can be regarded as a strengthening of the Belna's result.

Theorem 9. Let $f: A \rightarrow \mathbb{R}$, where $A$ is an arbitrary subset of $\mathbb{R}$. Then the set of points at which $f$ is symmetrically continuous but not continuous has the inner measure zero and contains no second category set having the Baire property.

Two corollaries below are strengthings of Corollary 8 .

Corollary 10. Let $E \subset \mathbb{R}$ be measurable and $f: E \rightarrow \mathbb{R}$ be symmetrically continuous. Then $f$ is continuous almost everywhere on $E$.

Corollary 11. Let $E \subset \mathbb{R}$ have the Baire property and $f: E \rightarrow \mathbb{R}$ be symmetrically continuous. Then $f$ is continuous at every point of $E$ except on a meager set.

In these corollaries the assumption that $E$ is measurable (has the Baire property) cannot be dropped, as the following example shows.

Example 3. Let $G_{1}$ be a group of full outer measure and let $G_{2}=\mathbb{Q}$. Then $f: G_{1} \cup G_{2} \rightarrow\{0,1\}$ defined like in Example 2 is symmetrically continuous (on its domain) but discontinuous everywhere on $G_{1} \cup G_{2}$. The same is true if $G_{1}$ is a group of second category.

Indeed, since both $G_{1}$ and $\mathbb{Q}$ are dense in $\mathbb{R}$, then $f$ must be discontinuous everywhere.

\section{Proof of the Main Result}

We give the proof of Theorem 9. Theorems 16 and 17 are technical and lengthy, but then the proof of Theorem 9 is short and easy.

We introduce two useful (though technical) definitions.

Definition 12. A symmetric covering relation on a set $E \subset \mathbb{R}$ is a family $V$ of closed intervals $[a, b]$ such that $\frac{a+b}{2} \in E$.

Definition 13. If $V$ is a symmetric cover relation on $E \subset \mathbb{R}$, then $V^{5}$ is a family of closed intervals $[a, b]$ such that there is a sequence of points $a=$ $x_{0}, x_{1}, \ldots, x_{5}=b$ such that $\left[x_{i}, x_{i+1}\right]$ belong to $V$ for $i=0,1,2,3,4$. We call the points $x_{1}, \ldots, x_{4}$ the intermediate endpoints. 
Uher [Uh] proved the following theorems (which have been reformulated by Thomson, and we give here Thomson's versions).

Theorem 14. (Uher [Th, thm. 3.25]) Let $E \subset \mathbb{R}$ have the Baire property and suppose $V$ is a symmetric cover relation on $E$ having the property that for every $x \in E$ there is a positive number $\delta(x)$ so that for every $t \in \mathbb{R}$

$$
0<t<\delta(x) \Rightarrow[x-t, x+t] \in V .
$$

Then there is an open set $G$ such that $E \backslash G$ is of the first category and every interval $[x-t, x+t]$ contained in $G$ belongs to $V^{5}$.

Theorem 15. (Uher [Th, thm. 3.26]) Let $E \subset \mathbb{R}$ be measurable and suppose $V$ is a symmetric cover relation on $E$ having the property that for every $x \in E$ there is a positive number $\delta(x)$ so that for every $t \in \mathbb{R}$

$$
0<t<\delta(x) \Rightarrow[x-t, x+t] \in V .
$$

Then, for almost all points $x \in E$ (in the sense of measure), there is a neighborhood $U_{x}$ of $x$ such that for each $x+t \in U_{x}$ the interval $[x, x+t]$ belongs to $V^{5}$.

We will prove two theorems that are strengthened versions of Uher theorems.

Theorem 16. Let $E \subset \mathbb{R}$ have the Baire property and suppose $V$ is a symmetric cover relation on $E$ having the property that for every $x \in E$ there is a positive number $\delta(x)$ so that for every $t \in \mathbb{R}$

$$
0<t<\delta(x) \Rightarrow[x-t, x+t] \in V .
$$

Then there is an open set $G$ such that $E \backslash G$ is of the first category and every interval $[x-t, x+t]$ contained in $G$ is in $V^{5}$. Moreover, the intermediate endpoints may be chosen from the set $E$.

Theorem 17. Let $E \subset \mathbb{R}$ be measurable and suppose $V$ is a symmetric cover relation on $E$ having the property that for every $x \in E$ there is a positive number $\delta(x)$ so that for every $t \in \mathbb{R}$

$$
0<t<\delta(x) \Rightarrow[x-t, x+t] \in V .
$$

Then, for almost all points $x \in E$ (in the sense of measure), there is a neighborhood $U_{x}$ of $x$ such that whenever $x+t \in U_{x}$, then the interval $[x, x+t]$ belongs to $V^{5}$. Moreover, the intermediate endpoints may be chosen from the set $E$. 
As we see, only the last parts of Theorems 16 and 17 make them stronger than their Uher/Thomson's counterparts. The structure of our proof is essentially identical to that presented in [Th] (attributed to Humke and Laczkovich). However, our proof is considerably more complicated from technical point of view.

Proof of Theorem 16. Let $\mathcal{I}$ denote the family of all open intervals $I$ with the property that for every $x, y \in I$ there is a chain $x=x_{0}, x_{1}, \ldots, x_{5}=y$ satisfying

$$
\left[x_{i}, x_{i+1}\right] \in V \text { for } i=0,1,2,3,4 \text { and } x_{i} \in E \text { for } i=1,2,3,4 .
$$

We will show that

if $E$ is residual in $(a, b)$, then there is a subinterval of $(a, b)$ belonging to $\mathcal{I}$.

The statement of the theorem then follows by taking for the set $G$ a union of a maximal pairwise disjoint subfamily of $\mathcal{I}$. Indeed any interval contained in $G$ is also contained in some interval from $\mathcal{I}$ so it satisfies the assertion of the theorem. To see that $E \backslash G$ is first category note that the set $E$ is residual in some nonempty open set $U$ for which $E \backslash U$ is meager. But then $U$ consists of disjoint intervals $(a, b)$ and $E$ is residual in each of them. So $G$ is dense in $U$ and, since it is open, it is residual in $U$. Thus $E \backslash G \subset(E \backslash U) \cup(U \backslash G)$ is meager as claimed.

To show (1) assume that $E$ is residual in $(a, b)$. For any positive $n \in \omega$ let

$$
E_{n}=\left\{x \in E: 0<t<\frac{1}{n} \Rightarrow[x-t, x+t] \in V\right\} .
$$

Then $E=\bigcup_{n=1}^{\infty} E_{n}$ and there is a number $n \in \omega$ so that $E_{n}$ is of second category in $(a, b)$. Thus there is an interval $I \subset(a, b)$ such that $E_{n}$ is of second category in every subinterval of $I$. Assume additionally that the length $|I|$ of $I$ is less than $\frac{1}{n}$. Take the interval $I^{\prime}$ concentric with $I$ and of the length $\frac{1}{10}|I|$. This is this interval $I^{\prime}$ that, as we shall show, belongs to $\mathcal{I}$.

Let $x, y \in I^{\prime}, x<y$. For the sake of simplicity assume that $x=0$. For $x \neq 0$ we repeat the construction below with the set $E$ replaced by $E-x$. Note that for any numbers $t$ with $|t|<\frac{3}{2}\left|I^{\prime}\right|=\frac{3}{20}|I|$ and $r$ with $\frac{1}{2} \leq|r| \leq 2$, the set

$$
r E_{n}+t \text { is second category in every subinterval of } I^{\prime} \text {. }
$$

To see this note that since $0 \in I^{\prime},[-0.9|I|, 0.9|I|] \subset I$. Since $|r| \geq \frac{1}{2}$ we have $[-0.45|I|, 0.45|I|] \subset r I$ and since $|t| \leq \frac{3}{2}\left|I^{\prime}\right|=0.15|I|,[-0.3|I|, 0.3|I|] \subset r I+t$. We see that the interval $I^{\prime}$ is contained in every interval $r I+t$. Since $E_{n}$ is of second category in every subinterval of $I$, so is $r E_{n}+t$ in $r I+t$. 
Similarly, the sets

$$
\pm \frac{1}{2} E+t \text { are residual in } I^{\prime} \text { for }|t|<\frac{3}{2}\left|I^{\prime}\right|
$$

We want to find a 5 -element chain $0=x_{0}, x_{1}, \ldots, x_{5}=y$ satisfying $(*)$. To do this we will find an interval $J \subset\left(\frac{9}{10} y, y\right)$ such that for every $z \in J$ there is a four element chain $0=x_{0}, x_{1}, \ldots, x_{4}=z$ satisfying

$$
\left[x_{i}, x_{i+1}\right] \in V \text { for } i=0,1,2,3 \text { and } x_{i} \in E \text { for } i=1,2,3 \text {. }
$$

This will finish the proof since then we select $z \in J$ such that $z$ is also in the set $Z=\left\{z \in\left(\frac{9}{10} y, y\right): z \in E\right.$ and $\left.[z, y] \in V\right\}$. The intersection of $J$ and $Z$ is nonempty since $\left(2 E_{n}-y\right) \cap E \cap\left(\frac{9}{10} y, y\right) \subset Z$ (as $z \in 2 E_{n}-y$ implies $\frac{z+y}{2} \in E_{n}$ so $[z, y] \in V)$ and, by $(2), 2 E_{n}-y$ is second category in every subinterval of $I^{\prime}$.

In the interval $\left(\frac{9}{10} y, y\right)$ the set $E$ is residual and, by $(2), E_{n}+\frac{3}{4} y$ is second category since $\frac{3}{4} y<\frac{3}{2}\left|I^{\prime}\right|$. Hence $E \cap\left(E_{n}+\frac{3}{4} y\right)$ is also second category in $\left(\frac{9}{10} y, y\right)$. It follows, from the fact that $E=\bigcup_{n=1}^{\infty} E_{n}$, that there is an integer $m$ and an open interval $J \subset\left(\frac{9}{10} y, y\right)$ so that the set every subinterval of $J$. We may assume that $|J|<\frac{1}{m}$.

To show that $J$ satisfies $(* *)$ pick $z \in J$. By (3) the set $\frac{1}{2} E+\frac{3}{4} y$ is residual in $J$ and, by (2), the set $\frac{1}{2} E_{n}+\frac{1}{4} z+\frac{3}{8} y$ is second category in $J$ since $\frac{3}{4} y, \frac{1}{4} z+\frac{3}{8} y<y<\left|I^{\prime}\right|$. Therefore we may choose a point

$$
t \in\left(\frac{1}{2} E+\frac{3}{4} y\right) \cap\left(\frac{1}{2} E_{n}+\frac{1}{4} z+\frac{3}{8} y\right) \cap J .
$$

Since $t \in \frac{1}{2} E+\frac{3}{4} y$, we have

$$
q=2 t-\frac{3}{2} y \in E
$$

Now we choose a point $s$ satisfying

$s \in\left(\frac{1}{2} E+\frac{3}{4} y\right) \cap\left(-\frac{1}{2} E+2 t-\frac{3}{4} y\right) \cap\left(\frac{1}{2} E+\frac{1}{2} z\right) \cap\left[E_{m} \cap\left(E_{n}+\frac{3}{4} y\right)\right] \cap J$ and $|s-t|<\frac{1}{2} \delta(q)$.

Such a point may be found since, by (3), the first three sets are residual in $J$, and the fourth one is of second category in every subinterval of $J$ so their intersection is dense in $J$. Denote by $p$ the point $p=s-\frac{3}{4} y$ and note that

$$
|q-2 p|=2|s-t|<\delta(q) \text { and } p \in E_{n} .
$$


Now define our points $x_{i}$ :

$$
\begin{aligned}
& x_{0}=0=x, \\
& x_{1}=2 p=2 s-\frac{3}{2} y \in E \text { since } 2\left(\frac{1}{2} E+\frac{3}{4} y\right)-\frac{3}{2} y=E, \\
& x_{2}=2 q-2 p=4 t-\frac{3}{2} y-2 s \in E \text { since } 4 t-\frac{3}{2} y-2\left(-\frac{1}{2} E+2 t-\frac{3}{4} y\right)=E, \\
& x_{3}=2 s-z \in E \text { since } 2\left(\frac{1}{2} E+\frac{1}{2} z\right)-z=E, \\
& x_{4}=z .
\end{aligned}
$$

So the second part of $(* *)$ is satisfied.

The centers of $\left[x_{i}, x_{i+1}\right]$ are:

$$
\begin{aligned}
& \frac{x_{0}+x_{1}}{2}=p \in E_{n} \text { by }(5), \\
& \frac{x_{1}+x_{2}}{2}=q \in E \text { by }(4), \\
& \frac{x_{2}+x_{3}}{2}=2 t-\frac{3}{4} y-\frac{1}{2} z \in E_{n} \text { since } t \in \frac{1}{2} E_{n}+\frac{1}{4} z+\frac{3}{8} y, \\
& \frac{x_{3}+x_{4}}{2}=s \in E_{m} .
\end{aligned}
$$

To finish the proof we only need to verify that each $\left[x_{i}, x_{i+1}\right]$ is in $V$. But this can be seen as follows. Intervals $\left[x_{0}, x_{1}\right]$ and $\left[x_{2}, x_{3}\right]$ are in $V$ since their centers are in $E_{n}$ and each $x_{i}$ is in the interval $I$ which has the length $<\frac{1}{n}$. The interval $\left[x_{1}, x_{2}\right] \in V$ since, by (5), $\left|x_{1}-\frac{x_{1}+x_{2}}{2}\right|=|2 p-q|<\delta(q)$. And finally $\left[x_{3}, x_{4}\right]$ belongs to $V$ because $\left|x_{4}-s\right|=|z-s|<\frac{1}{m}$ as both $s, z \in J$ which has length less than $\frac{1}{m}$.

In the proof of the next theorem we use the following notions and facts. By $|A|$ we denote the outer (Lebesgue) measure of the set $A, d(A)$ denotes the set of points of (outer) density of $A$, i.e., the set $\left\{x \in \mathbb{R}: \lim _{h \rightarrow 0^{+}} \frac{|A \cap[x-h, x+h]|}{2 h}=1\right\}$. The well known theorem of Lebesgue (see e.g. [Th, thm. A.11]) says that for any set $A$ almost all points of $A$ are its points of density. $d(A)$ is always a measurable set (even for nonmeasurable $A$ ) and $d(d(A))=d(A)$. If $x \in d(A)$, then $a x+b \in d(a A+b)$ for $a, b \in \mathbb{R}, a \neq 0$. In particular if $A$ consists only of its density points, then so does $a A+b$ for $a, b \in \mathbb{R}, a \neq 0$. We also use the following lemma. 
Lemma 18. [Th, thm. A.11] If sets $A_{n} \subset \mathbb{R}$, where $n \in\{1,2, \ldots, k\}$, are all measurable except at most one of them and $z$ is a point of density of each of them, then $z$ is also a point of density of their intersection. In particular in every neighborhood of $z$ there is a point of $\bigcap_{n=1}^{k} A_{n}$.

Proof of Theorem 17 . The proof follows the pattern of the proof of Thm. 16; we will refer to the conditions $(*)$ and $(* *)$ from that proof.

We assume throughout the proof that every measurable set consists only of its density points. Let

$$
E_{n}=\left\{x \in E: 0<t<\frac{1}{n} \Rightarrow[x-t, x+t] \in V\right\} .
$$

We show that every point that belongs to the set $\bigcup_{n=1}^{\infty} d\left(E_{n}\right)$ has a neighborhood satisfying the assertion of the theorem. Thus almost every point of $E$ satisfies it since $E \backslash \bigcup_{n=1}^{\infty} d\left(E_{n}\right)$ is of measure 0 .

Let $x$ be a point of density of $E_{n}$. Assume for notational convenience that $x=0$ (for if $x \neq 0$, then replace the set $E$ by $E-x$ ). There is a number $\eta, 0<\eta<\frac{1}{n}$, such that for every $y$ with $|y|<\eta$ and every $t$ with $|t|<2|y|$ each of the sets

$$
2 E_{n}+t, \pm \frac{1}{2} E_{n}+t, \frac{3}{2} E_{n}+t, \text { and } E \cap\left(E_{n}+t\right)
$$

intersects the interval $\left(\frac{9}{10} y, y\right)$ in a set of outer measure greater than $\frac{9}{100}|y|$. We may take $\eta=\frac{1}{6} \delta$, where $\delta$ is such that for every $0<h<\delta$ we have $\frac{\left|E_{n} \cap[-h, h]\right|}{2 h} \geq \frac{999}{1000}$. To see that this $\eta$ works take for example the set $\frac{1}{2} E_{n}+t$ and note that $\left(\frac{1}{2} E_{n}+t\right) \cap(0.9 y, y)=\frac{1}{2}\left[E_{n} \cap(1.8 y-2 t, 2 y-2 t)\right]+t$. Numbers $|1.8 y-2 t|$ and $|2 y-2 t|$ are less than $6|y|<\delta$, so

$$
\begin{aligned}
\left|E_{n} \cap(1.8 y-2 t, 2 y-2 t)\right| & =\mid E_{n} \cap(-6 y, 6 y) \\
& \backslash\left[E_{n} \cap[(-6 y, 6 y) \backslash(1.8 y-2 t, 2 y-2 t)]\right] \mid \\
& \geq \frac{999}{1000} 12|y|-(12|y|-0.2|y|) \\
& =\frac{188}{1000}|y|>2 \frac{9}{100}|y| .
\end{aligned}
$$

The calculations for other sets in (6) are similar.

Fix any $y \in(0, \eta)$. We prove that there is a chain $0=x_{0}, x_{1}, \ldots, x_{5}=y$ satisfying $(*)$. A similar argument would work for $y<0$.

Let $Z=\left\{z \in\left(\frac{9}{10} y, y\right):[z, y] \in V\right\}$. Note that $\left(2 E_{n}-y\right) \cap\left(\frac{9}{10} y, y\right) \subset Z$ and so, by (6), $|Z| \geq \frac{9}{100} y$. To prove the theorem it is enough to show that there is a measurable set $F \subset\left(\frac{9}{10} y, y\right) \cap E$ such that $|F|>\frac{1}{100} y$ and that 
for every $z \in F$ there is a four element chain $0=x_{0}, \ldots, x_{4}=z$ satisfying (**). Indeed if this were true, then $F \cap Z \neq \emptyset$ and choosing any $z \in F \cap Z$ we would obtain a four element chain which expanded by $x_{5}=y$ would give us our desired chain.

Recall that, by (6), $\left.\mid E \cap\left(E_{n}+\frac{3}{4} y\right)\right) \cap\left(\frac{9}{10} y, y\right) \mid>\frac{9}{100} y$, so from the fact that $E$ is an increasing sum of $E_{m}$ 's, there is an integer $m$ so that

$$
\left|E_{m} \cap\left(E_{n}+\frac{3}{4} y\right) \cap\left(\frac{9}{10} y, y\right)\right|>\frac{8}{100} y .
$$

Denote $A_{m}=E_{m} \cap\left(E_{n}+\frac{3}{4} y\right) \cap\left(\frac{9}{10} y, y\right)$ and write

$$
F=\left(\frac{1}{2} E+\frac{3}{4} y\right) \cap d\left(\frac{2}{3} E_{n}+\frac{1}{2} y\right) \cap d\left(A_{m}\right) \cap E .
$$

We show that all points of $F$ have the property claimed for it.

First note that, by (6) and (7),

$$
|F|>\frac{1}{10} y-\frac{1}{100} y-\frac{1}{100} y-\frac{2}{100} y-\frac{1}{100} y>\frac{5}{100} y .
$$

Let $z \in F$. So $z$ is a density point of $F$. Since $z \in d\left(\frac{2}{3} E_{n}+\frac{1}{2} y\right)$ it follows that $z$ belongs to $d\left(\frac{1}{2} E_{n}+\frac{1}{4} z+\frac{3}{8} y\right)$ (indeed $\frac{3}{4} z \in d\left(\frac{3}{4}\left(\frac{2}{3} E_{n}+\frac{1}{2} y\right)\right)$ so $\frac{1}{4} z+\frac{3}{4} z \in$ $\left.d\left(\frac{3}{4}\left(\frac{2}{3} E_{n}+\frac{1}{2} y\right)+\frac{1}{4} z\right)\right)$, and since $z \in E, z \in \frac{1}{2} E+\frac{1}{2} z$.

We may pick a point $t$ so that

$$
t \in\left(\frac{1}{2} E_{n}+\frac{1}{4} z+\frac{3}{8} y\right) \cap\left(\frac{1}{2} E+\frac{1}{2} z\right) \cap\left(\frac{1}{2} E+\frac{3}{4} y\right) \cap d\left(A_{m}\right)
$$

and $|z-t|<\frac{1}{m}$.

This is possible since by Lemma 18 since all sets above, except possibly the first one, are measurable and the point $z$ is a density point of each of them.

We define $q=2 t-\frac{3}{2} y$ and note that $q \in E$ since $2\left(\frac{1}{2} E+\frac{3}{4} y\right)-\frac{3}{2} y=E$. Note also that $t$ is a density point of the set $-\frac{1}{2} E+2 t-\frac{3}{4} y$ as $t \in \frac{1}{2} E+\frac{3}{2} y$.

We now pick a point $s$. We want $s$ to satisfy:

$$
\begin{gathered}
s \in\left(\frac{1}{2} E+\frac{1}{2} z\right) \cap\left(\frac{1}{2} E+\frac{3}{4} y\right) \cap\left(-\frac{1}{2} E+2 t-\frac{3}{4} y\right) \cap A_{m}, \\
|s-t|<\frac{1}{2} \delta(q) \text { and }|s-z|<\frac{1}{m} .
\end{gathered}
$$

Once again by Lemma 18 there exists a point $s$ being in all four sets and arbitrary close to $t$ ( $t$ is a density point of each of the above set). Taking $s$ 
close enough to $t$ we guarantee the last condition since $|s-z|<|z-t|+|t-s|$ and $|z-t|<\frac{1}{m}$.

Define the point $p=s-\frac{3}{4} y$ and note that $|q-2 p|=2|s-t|<\delta(q)$. The points $x_{0}, x_{1}, \ldots, x_{4}$ satisfying $(* *)$ are defined as follows:

$$
\begin{aligned}
& x_{0}=x=0 \\
& x_{1}=2 p=2 s-\frac{3}{2} y \in E \text { since } 2\left(\frac{1}{2} E+\frac{3}{4} y\right)-\frac{3}{2} y=E \\
& x_{2}=2 q-2 p=4 t-2 s-\frac{3}{2} y \in E \text { since } 4 t-\frac{3}{2} y-2\left(-\frac{1}{2} E+2 t-\frac{3}{4} y\right)=E, \\
& x_{3}=2 s-z \in E \text { since } 2\left(\frac{1}{2} E+\frac{1}{2} z\right)-z=E \\
& x_{4}=z
\end{aligned}
$$

The centers of the intervals $\left[x_{i}, x_{i+1}\right]$ are:

$$
\begin{aligned}
& \frac{x_{0}+x_{1}}{2}=p=s-\frac{3}{4} y \in E_{n} \text { since } s \in A_{m} \subset E_{n}+\frac{3}{4} y . \\
& \frac{x_{1}+x_{2}}{2}=q=2 t-\frac{3}{2} y \in E \text { since } t \in \frac{1}{2} E+\frac{3}{4} y . \\
& \frac{x_{2}+x_{3}}{2}=2 t-\frac{3}{4} y-\frac{1}{2} z \in E_{n} \text { since } t \in \frac{1}{2} E_{n}+\frac{1}{4} z+\frac{3}{8} y . \\
& \frac{x_{3}+x_{4}}{2}=s \in E_{m} \text { since } s \in A_{m} \subset E_{m} .
\end{aligned}
$$

Intervals $\left[x_{0}, x_{1}\right]$ and $\left[x_{2}, x_{3}\right]$ are in $V$ since their centers are in $E_{n}$ and each $x_{i}$ is in the interval $I$ which has the length $<\frac{1}{n}$. The interval $\left[x_{1}, x_{2}\right] \in V$ since $\left|x_{1}-\frac{x_{1}+x_{2}}{2}\right|=\left|x_{1}-q\right|=2|s-t|<\delta(q)$. And finally $\left[x_{3}, x_{4}\right]$ belongs to $V$ because $\left|x_{4}-s\right|=|z-s|<\frac{1}{m}$. This way we see that each interval $\left[x_{i}, x_{i+1}\right]$ belongs to $V$ and the proof is finished.

Proof of Theorem 9. Let us denote by $S C_{f}$ the set of points where $f$ is symmetrically continuous and by $C_{f}$ the set of continuity points. We prove only that $S C_{f} \backslash C_{f}$ contains no measurable set of positive measure. The proof that $S C_{f} \backslash C_{f}$ contains no second category set having the Baire property is essentially the same.

Assume that $E \subset S C_{f}$ is measurable. We will show that almost all points in $E$ are also in $C_{f}$. This shows that the only measurable subsets of $S C_{f} \backslash C_{f}$ are of measure zero so $S C_{f} \backslash C_{f}$ has inner measure zero.

Fix an $\varepsilon>0$ and define a symmetric covering relation on $E$ :

$$
V=\{[x-t, x+t]: x \pm t \in E \Rightarrow|f(x-t)-f(x+t)|<\varepsilon\} .
$$


This relation satisfies assumption of Theorem 17 . So for almost every $x \in E$ there is a neighborhood $U_{x}$ so that $x+t \in U_{x} \Rightarrow[x, x+t] \in V^{5}$ and intermediate endpoints are in $E$, that is there are points $x_{0}=x, x_{1}, \ldots, x_{5}=x+t$ such that $x_{i} \in E$ for $i=1,2,3,4$ and $\left[x_{i}, x_{i+1}\right] \in V$ for $i=0, \ldots, 4$. Therefore $|f(x)-f(x+t)|<5 \varepsilon$. Since $\varepsilon$ has been chosen arbitrarily, $f$ is continuous in almost all points of $E$.

\section{Extensions of Continuous and Symmetrically Contin- uous Functions}

In this section we look at the extension properties of symmetrically continuous functions and compare them with these properties for continuous functions.

We know that every continuous function $f: A \rightarrow \mathbb{R}$ defined on a subset of $\mathbb{R}$ can be extended to a continuous function defined on a dense $G_{\delta}$ set. This set can be of course residual. Note that continuity distinguishes between measure and category as it is possible to find function that can be only extended to a measure zero $G_{\delta}$ set. We will see in Theorem 20 that some symmetrically continuous functions cannot be extended beyond measure zero and first category domain.

If we look at Example 3 we see that symmetrically continuous functions defined there is discontinuous on its domain so on a full outer measure or second category set. Thus, by Corollaries 10 and $11, f$ cannot be extended to a symmetrically continuous function defined on a measurable set or on a set having the Baire property.

For an example of a similar function $f$ defined on a symmetric domain see Section 5. In Theorem 20 we give much stronger example in which domain may be chosen to be of measure zero and first category.

A different question is whether having a function $f: A \rightarrow \mathbb{R}$ that is continuous (or symmetrically continuous) we may extend it to the whole real line leaving it continuous (or symmetrically continuous) on the set $A$. For continuous functions as the next theorem shows this is true.

Fact 19. If $f: A \rightarrow \mathbb{R}$ is continuous, then there is an extension $\hat{f}$ of $f$ defined on the whole $\mathbb{R}$ with $A \subset C_{\hat{f}}$, where $C_{\hat{f}}$ is the set of points of continuity of $\hat{f}$.

Proof. If $A$ is not dense in $\mathbb{R}$, then $\mathbb{R} \backslash \operatorname{cl}(A)$ consists of disjoint intervals $(a, b)$ and we extend our function by defining $f$ on each of them as a continuous function joining $\sup _{x \rightarrow a, x \in A} f(x)$ with $\sup _{x \rightarrow b, x \in A} f(x)$ (even if one of them or both are infinity). So we may assume that $A$ is dense in $\mathbb{R}$.

Assume first that $f$ is bounded. In this case we may assume that $f: A \rightarrow$ $[0,1]$. Take a closure $\operatorname{cl}(f)$ of the function $f$ in $\mathbb{R}^{2}(f$ is a set of pairs and thus 
a subset of $\left.\mathbb{R}^{2}\right)$. Since $A$ is dense, the projection $\pi(\operatorname{cl}(f))$ of $\operatorname{cl}(f)$ onto the first coordinate is the whole real line. This is so, as for every finite interval $[a, b]$, the set $\operatorname{cl}\left(\left.f\right|_{[a, b]}\right)$ is compact and its projection is also compact, thus contains $A \cap[a, b]$ - a dense set in $[a, b]$.

Let $I_{x}=\{y:\langle x, y\rangle \in \operatorname{cl}(f)\}$. Then every $I_{x}$ is nonempty. Also, for every $x \in A$ the set $I_{x}$ is the one element set $\{f(x)\}$. Indeed, for every $\left\{\left\langle x_{n}, f\left(x_{n}\right)\right\rangle\right\}$ in $f$ with $\left\{x_{n}\right\}$ converging to $x$, we have $\lim f\left(x_{n}\right)=f(x)$ by the continuity of $f$. Take an indexed selector $S$ of the family $\left\{I_{x}: x \in \mathbb{R}\right\}$ (i.e., $S$ is a set of pairs $\langle x, y\rangle$, where $\left.y \in I_{x}\right)$. Then $S$ is a function from $\mathbb{R}$ to $[0,1]$ and $S$ agrees with $f$ on every $x \in A$. It is easy to check that $S$ is continuous in every point of $A$.

If $f$ is unbounded, then take the function $g=\arctan \circ f$, which is bounded. By the continuity of arctan we have that $g$ is continuous in every point where $f$ is continuous.

Having an extension $\hat{g}$ of $g$, we come back to $f$ taking $\hat{f}=\tan \circ \hat{g}$, where we define $\hat{f}\left( \pm \frac{\pi}{2}\right)=0$. Once again by the continuity of the function tan we preserve points of continuity of $\hat{g}$.

Once again the translation of the above theorem into symmetrical language fails to hold.

Theorem 20. There is a function $f: A \rightarrow\{0,1\}$ that is symmetrically continuous everywhere on $A$ with the following property: if $F$ is an extension of $f$ to a domain $D$ such that $D$ has positive measure or $D$ is of second category with the Baire property, then $F$ is not symmetrically continuous at some point on $A$.

Proof. We will construct inductively (using transfinite induction) a set $A$ and will define function $f$ on $A$. Function $f$ will have two properties: for every perfect set $P$, there is a point $x \in P$ such that there is a pair of sequences $\left\{y_{n}\right\}$ and $\left\{z_{n}\right\}$ in $A$ converging to $x$ and symmetric about $x$, (i.e. $\frac{y_{n}+z_{n}}{2}=x$ for all $n) ; f$ will have value 1 for all $y_{n}^{\prime} s$ and value 0 for all $z_{n}^{\prime} s$. Then, of course, $x$ cannot belong to the domain of any symmetrically continuous extension of $f$ and, since any measurable set of positive measure or Baire measurable set of second category contains a perfect set, $f$ cannot be extended to any of these sets.

Let $\left\{P_{\alpha}: \alpha<\mathbf{c}\right\}$ be a list of all perfect subsets of $\mathbb{R}$. Take any point $x_{0} \in P_{0}$ and define $y_{n}^{0}=x_{0}-\frac{1}{n}$ and $z_{n}^{0}=x_{0}+\frac{1}{n}$. Put also $f_{0}:\left\{y_{n}^{0}, z_{n}^{0}: n \in \omega\right\} \rightarrow\{0,1\}$, $f_{0}\left(y_{n}^{0}\right)=1, f_{0}\left(z_{n}^{0}\right)=0$. (We do not define $f_{0}\left(x_{0}\right)$.)

Since $x_{0}$ is the only accumulation point of the set $\left\{y_{n}^{0}, z_{n}^{0}: n \in \omega\right\}$, the function $f_{0}$ is symmetrically continuous (vacuously) on its domain. Denote by 
$T_{0}$ the linear space over $\mathbb{Q}$ spanned by the set $\left\{y_{n}^{0}, z_{n}^{0}: n \in \omega\right\}$ and note that $x_{0} \in T_{0}$.

Assume that for $\beta<\alpha$ we have defined points $x_{\beta} \in P_{\beta}$ and sequences $\left\{y_{n}^{\beta}\right\}$ and $\left\{z_{n}^{\beta}\right\}$ converging to $x_{\beta}$ and $\frac{y_{n}^{\beta}+z_{n}^{\beta}}{2}=x_{\beta}$. Assume also that $x_{\beta}, y_{n}^{\beta}, z_{n}^{\beta} \notin T_{\beta}$ where $T_{\beta}=$ linear space over $\mathbb{Q}$ spanned by the set $\left\{x_{\gamma}, y_{n}^{\gamma}, z_{n}^{\gamma}: n \in \omega, \gamma<\beta\right\}$ (i.e. the points $x_{\beta}, y_{n}^{\beta}, z_{n}^{\beta}$ are independent of the previous ones).

Functions $f_{\beta}:\left\{y_{n}^{\beta}, z_{n}^{\beta}: n \in \omega\right\} \rightarrow\{0,1\}$ are defined like $f_{0}$; that is, $f_{\beta}\left(y_{n}^{\beta}\right)=$ 1 and $f_{\beta}\left(z_{n}^{\beta}\right)=0$. (We leave $f_{\beta}\left(x_{\beta}\right)$ not defined.)

Let $T_{\alpha}$ be the linear space over $\mathbb{Q}$ spanned by the set $\left\{x_{\beta}, y_{n}^{\beta}, z_{n}^{\beta}: n \in\right.$ $\omega, \beta<\alpha\}$. The space $T_{\alpha}$ has cardinality $|\alpha| \times \omega<\mathbf{c}$ and we may find a point $x_{\alpha} \in P_{\alpha} \backslash T_{\alpha}$ and sequences $\left\{y_{n}^{\alpha}\right\},\left\{z_{n}^{\alpha}\right\}$ disjoint with $T_{\alpha}$, converging to $x_{\alpha}$, and with $\frac{x_{n}^{\alpha}+z_{n}^{\alpha}}{2}=x_{\alpha}$ for all $n \in \omega$. As before we define $f_{\alpha}\left(y_{n}^{\alpha}\right)=0$ and $f_{\alpha}\left(z_{n}^{\alpha}\right)=1$. Again $f_{\alpha}$ is (vacuously) symmetrically continuous on its domain.

Having our construction done for all $\alpha<\mathbf{c}$ we define $f=\bigcup_{\alpha<\mathbf{c}} f_{\alpha}$. Since points $y_{n}^{\alpha}$ and $z_{n}^{\alpha}$ are linearly independent of the points $y_{n}^{\beta}$ and $z_{n}^{\beta}$ for $\alpha \neq \beta$, the function $f$ is vacuously symmetrically continuous. Putting $A=\operatorname{dom}(f)=$ $\left\{y_{n}^{\alpha}, z_{n}^{\alpha} ; n \in \omega, \alpha<\mathbf{c}\right\}$ we have constructed the desired set and the function defined on it.

It is worth to mention that we may strengthen the theorem above by adding that $A$ may be of measure zero and first category. (We may select points $y_{n}^{\alpha}$ and $z_{n}^{\alpha}$ from the set $C$ constructed in [Sz, Col.10].)

\section{$5 \quad$ Functions on Symmetric Domains}

Definition 1 may seem a bit strange since we do not require both of the points $x+h$ and $x-h$ to be in the domain. Most of our counterexamples have nonsymmetric domain and the functions are vacuously symmetrically continuous. Here we try to justify our definition by showing that adding symmetry to the domain does not give us any stronger properties that functions on nonsymmetric domains do not possess.

We say that a set $A \subset \mathbb{R}$ is symmetric if

$$
\forall \forall_{x \in A} \forall_{h} \quad x+h \in A \Longleftrightarrow x-h \in A .
$$

For example every additive group in $\mathbb{R}$ is symmetric.

\section{Example 4. $L$}

et $A=\left\{\frac{k}{3^{n}}: k \in \mathbb{Z}, n \in \omega\right\}$ and $f: A \rightarrow\{0,1\}$ be defined by

$$
f\left(\frac{k}{3^{n}}\right)= \begin{cases}0 & \text { when } \mathrm{k} \text { is even } \\ 1 & \text { when } \mathrm{k} \text { is odd. }\end{cases}
$$


Then $f$ is symmetrically continuous and $A$ is symmetric yet $f$ is discontinuous everywhere on its domain.

Proof. $A$ is a group so is obviously symmetric. It is immediate that the symmetric reflection of any point $\frac{k}{3^{n}}$ about a point $\frac{l}{3^{m}}$ preserves the parity of the numerator $\left(2 \frac{l}{3^{m}}-\frac{k}{3^{n}}=\frac{2 l 3^{n}-k 3^{m}}{3^{m+n}}\right)$. Since both $f^{-1}(0)$ and $f^{-1}(1)$ are dense, $f$ is discontinuous everywhere.

Example 5. Let $H$ be a Hamel base containing 1 and let $S$ be linear space over $\mathbb{Q}$ spanned by $H \backslash\{1\}$. Define $A=\{x+k: x \in S, k \in \mathbb{Z}\}$ and $f: A \rightarrow\{0,1\}$ by

$$
f(x+k)= \begin{cases}0 & \text { when } k \text { is even } \\ 1 & \text { when } k \text { is odd }\end{cases}
$$

Then $f$ is symmetrically continuous on $A, A$ is symmetric, and yet $f$ is discontinuous everywhere on $A$. Moreover $f$ cannot be extended to a symmetrically continuous function defined on any measurable set or a set with the Baire property.

Proof. Just as in the previous example, $A$ is a group, thus symmetric, and $f$ is symmetrically continuous. (We preserve the parity of $k$ in reflections.) Both $f^{-1}(1)$ and $f^{-1}(0)$ are dense in $\mathbb{R}$ so $f$ is discontinuous. Moreover $f$ cannot be extended to any measurable (or having the Baire property) domain being symmetrically continuous on the greater domain as that would imply continuity almost everywhere (on a residual set) on its domain (Corollaries 10 and 11).

The function $f$ above cannot be even "almost extended" (in a sense that the extension differs from $f$ only on a set of measure 0 ) to a symmetrically continuous function. This follows from the fact that the sets $f^{-1}(1)$ and $f^{-1}(0)$ are nonmeasurable. Note that all rational translations of $f^{-1}(1)$ and $f^{-1}(0)$ cover $\mathbb{R}$ so these sets have positive outer measure. So even upon removing sets of measure zero from them they remain dense in $\mathbb{R}$.

As we see, symmetric domains when nonmeasurable do not improve regularity of symmetrically continuous functions defined on them. This is a strong argument in favor of our definition (Definition 1) since we do not need to distinguish between symmetric and nonsymmetric domains and we are still able to get positive results (e.g. Corollaries 10 and 11). 


\section{References}

[Be] C. L. Belna, Symmetric continuity of real functions, Proc. Amer. Math. Soc. 87 (1983), 99-102.

[Ci] K. Ciesielski, Set Theory for the Working Mathematician, London Math. Soc. Student Texts 39, Cambridge Univ. Press 1997.

[Fr] H. Fried, Über die symmetrische Stetigkeit von Funktionen, Fund. Math. 29 (1937), 134-137.

[L] L. Larson, Symmetricreal analysis:a survey, Real Anal. Exchange 9 (1983/84), 154-178.

[Pe] I. N. Pesin, On the measurability of symmetrically continuous functions, Teor. Funkcii Funkktsional. Anal. i Prilozen 5 (1967), 99-101.

[Pr] D. Preiss, A note on symmetrically continuous functions, Časopis Pěst. Mat. 96 (1971), 262-264.

[SZ] E. M. Stein, A. Zygmund, On the differentiability of functions, Studia Math. 23 (1960), 295-307.

[Sz] M. Szyszkowski, Points of weak symmetric continuity, Real Anal. Exchange 24 (1998/99), 807-813.

[Th] B. S. Thomson, Symmetric properties of real functions, Marcel Dekker, 1994.

[Uh] J. Uher, Symmetric continuity implies continuity, Trans. Amer. Math. Soc. 293 (1986), 421-429. 